\title{
A GEOMETRIA FRACTAL DA REDE DE DRENAGEM DA BACIA HIDROGRÁFICA DO CAETÉ, ALFREDO WAGNER-SC ${ }^{1}$
}

\author{
Leandro Redin Vestena² e Masato Kobiyama ${ }^{3}$
}

\begin{abstract}
RESUMO - Os objetivos deste trabalho foram estimar e avaliar a dimensão fractal da rede de drenagem da bacia hidrográfica do Caeté, em Alfredo Wagner, SC, a partir de diferentes métodos, com o propósito de caracterizar as formas geomorfológicas irregulares. A rede de drenagem apresenta propriedades multifractais. As dimensões fractais para os segmentos individuais (df) e para a rede de drenagem inteira (Df) foram determinadas por métodos que se fundamentaram nas razões de Horton e pelo método da contagem de caixas (Box-Counting). A rede de drenagem tem característica de autoafinidade. A dimensão fractal proveniente da relação de parâmetros obtidos pelas Leis de Horton apresentou resultados dentro dos limiares da teoria da geometria fractal.
\end{abstract}

Palavras-chave: Morfometria, Dimensão fractal e Rio Caeté.

\section{FRACTAL GEOMETRY OF THE DRAINAGE NETWORK OF THE CAETÉ RIVER WATERSHED, ALFREDO WAGNER-SC}

\begin{abstract}
The objective of the present work was to evaluate the fractal dimensions of the drainage network of the Caeté river watershed, Alfredo Wagner/SC, with different methods in order to characterize the irregular geomorphologic forms. The drainage network possesses multi-fractal properties. That is why the fractal dimensions for the individual segments ( $d f$ ) and for the entire network $(D f)$ were evaluated with Horton's Laws and the Box-Counting method. The drainage network has self-affinity characteristics. The fractal dimension obtained through the parameters relationship of Horton's Laws showed the results within the thresholds of the fractal geometry theory.
\end{abstract}

Keywords: Morphometry, Fractal dimensions and Caeté river.

\section{INTRODUÇÃO}

A geometria fractal foi introduzida por Mandelbrot (1983) para descrever padrões de distribuição, estrutura e dinamismo da natureza significativamente complexos, ou seja, não lineares, irregulares, caóticos e aleatórios.

Uma das ferramentas propostas para a análise dos sistemas complexos é a dimensão fractal, definida como a medida do grau de irregularidades em diferentes escalas; e é relacionada com o aumento da medida de um objeto, enquanto a escala do instrumento de medida diminui.

A alteração na escala de observação dos fractais determina uma quantidade de elementos similares que ocorrem também de formas diferentes. A sequência de alteração estabelece a noção de escalonamento, que pode ser analisada como a relação entre o tamanho e a quantidade de ocorrências nos diversos níveis de observação, conforme destacou Christofoletti (1997). A dimensão fractal é o valor do expoente do escalonamento, relacionando o número de ocorrências desses elementos com a categoria de seus diversos tamanhos. Assim, pode-se dizer que um objeto com menor dimensão fractal é menos complexo do que um objeto com maior dimensão fractal.

As bacias de drenagem, segundo Nikora (1994), apresentam estrutura de multiescalonamento fractal e dimensão autossimilar. O multiescalonamento demonstra que as redes de drenagem possuem detalhes semelhantes

\footnotetext{
${ }^{1}$ Recebido em 25.05.2008 e aceito para publicação em 02.03.2010.

${ }^{2}$ Universidade Estadual do Centro-Oeste, UNICENTRO, Brasil. E-mail: <lvestena@gmail.com>.

${ }^{3}$ Universidade Federal de Santa Catarina, UFSC, Brasil. E-mail: <kobiyama@ens.ufsc.br>.
} 
em escalas diferentes e que a autossimilaridade com o padrão geométrico é repetida nas diversas escalas de grandeza espacial de observação.

Uma bacia de drenagem inclui em suas transformações bacias menores ao longo de seus afluentes. Na natureza, esse procedimento termina com as bacias de drenagem de primeira ordem, cujos tamanhos determinam a fractalidade da escala interna.

A dimensão fractal da rede de drenagem é importante por caracterizar as propriedades da escala e indicar a associação entre medida e escala, visto que as medidas geomorfológicas, o comprimento dos cursos fluviais, a densidade de drenagem e a declividade são tipicamente mensurados de mapas e usados no planejamento do manejo da bacia hidrográfica.

Na bacia hidrográfica do rio Caeté, localizada no Município de Alfredo Wagner, SC, a paisagem é representada por escarpas, interrompidas por patamares e por um relevo fortemente dissecado, com encostas íngremes. A rede de drenagem na bacia apresenta forma bem irregular. A densidade de drenagem é significativa, representada por grande número de cursos fluviais $\left(1,949 \mathrm{~km} / \mathrm{km}^{2}\right)$. A grande diferença altimétrica reflete-se em cursos fluviais encachoeirados, com saltos e corredeiras que favorecem os processos de transporte de sedimentos (VESTENA et al., 2006).

De acordo com Tonello et al. (2006), o padrão de drenagem afeta a disponibilidade de sedimento e a taxa de formação do deflúvio. Assim, é preciso expressar quantitativamente as características de forma, de processos e de suas inter-relações, pois esses parâmetros podem revelar indicadores físicos específicos de determinada bacia hidrográfica, de forma a qualificarem as alterações ambientais.

A análise morfométrica, a partir da aplicação das Leis de Horton, tem sido tradicionalmente utilizada na caracterização geomorfológica das bacias hidrográficas, porém, com o surgimento da geometria fractal para estudar formas irregulares, novos caminhos surgiram para a compreensão e entendimento das formas irregulares das redes de drenagem. Nesse sentido, destacam-se, entre outros, os trabalhos de Goodchild (1982), Tarboton et al. (1988), Veltri et al. (1996), Agnese et al. (1996), Puente e Castillo (1996), Tarboton (1996), Roth et al. (1996), Schüller et al. (2001), Kobiyama e Bueno Junior (2002) e Bueno Junior (2002).
Este trabalho teve por objetivo determinar a dimensão fractal da rede de drenagem e dos segmentos dos cursos fluviais da bacia hidrográfica do rio Caeté. A dimensão fractal assume relevante importância na compreensão da dinâmica dos processos hidrológicos e na evolução das redes de drenagem (bacia hidrográfica), uma vez que o comportamento hidrológico em uma bacia hidrográfica é influenciado, principalmente, pela interação das suas características hidrogeomorfológicas (forma, relevo, área, rede de drenagem e densidade de drenagem, entre outros).

\section{CARACTERIZAÇÃO DA ÁREA DE ESTUDO}

A bacia hidrográfica do Caeté, com aproximadamente 163 km² de área, localiza-se no Município de Alfredo Wagner, região serrana do Estado de Santa Catarina, entre as coordenadas geográficas $27^{\circ} 41^{\prime} 41^{\prime \prime}$ a $27^{\circ} 52^{\prime} 36^{\prime}$ ” de latitude sul e $49^{\circ} 11^{\prime} 15^{\prime \prime}$ ' a $49^{\circ} 20^{\prime} 48^{\prime \prime}$ de longitude oeste (coordenadas Universal Transversa de Mercator - UTM: 6935296 a 6915356 e 678741 a 662769 , respectivamente), no datum SAD 69 (South American Datum -1969) (Figura 1).

O rio Caeté é formado pelos rios Santo Anjo e Perito, com nascentes em altitudes de 1.140 e 1.600 m, respectivamente. O clima predominante é o mesotérmico úmido, de acordo com a classificação de Köppen, com verões frescos e temperaturas anuais médias entre $16^{\circ} \mathrm{C}$ e $20^{\circ} \mathrm{C}$, inverno frio, com a ocorrência de geadas e verão amenizado pelas altitudes. As chuvas são abundantes e distribuídas ao longo do ano, com precipitações anuais em torno de $1.700 \mathrm{~mm}$ (SANTA CATARINA, 1986).
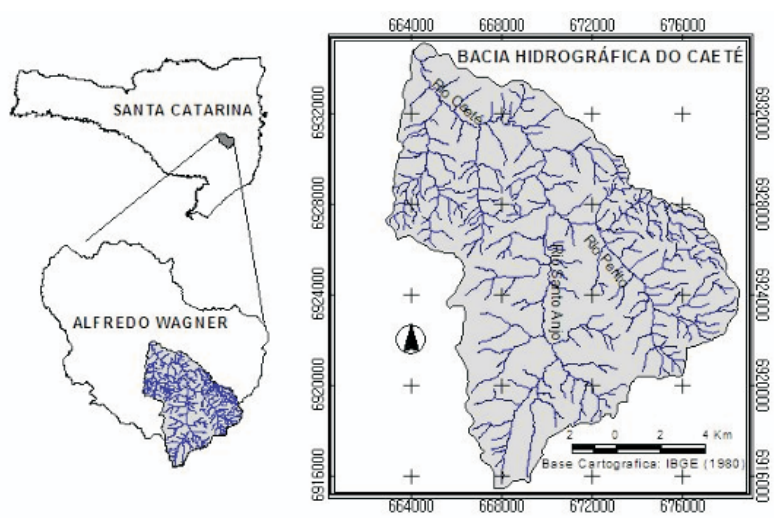

Figura 1 - Localização da bacia hidrográfica do Caeté, no Município de Alfredo Wagner, SC.

Figure 1 - Location of the Caeté watershed in Alfredo Wagner City, the State of Santa Catarina, Brazil. 
O relevo é dissecado com encostas íngremes, patamares e vales estruturais, onde atividades de uso inadequadas do solo na bacia tornam mais suscetíveis às ocorrências dos processos erosivos, como destacaram Checchia et al. (2004) e Silva e Kobiyama (2004). A dissecação é representada por forma de relevo do tipo montanhoso, escarpado, colinoso e morraria.

O uso da terra é caracterizado por matas, reflorestamento, campo, solos expostos e cultivos. Segundo o mapeamento temático das classes de uso da terra, realizado por Viecili (2005), na bacia do rio Caeté as áreas com mata predominam em 52,2\%, seguidas por $41,8 \%$ de campo, $2,2 \%$ de solos expostos, $2,1 \%$ de reflorestamento e $1,7 \%$ de cultivos. A principal atividade agrícola é o cultivo da cebola, associada geralmente com lavouras de feijão e milho, em pequenas propriedades.

\section{MATERIAL E MÉTODOS}

A base cartográfica foi obtida a partir das cartas topográficas (Tabela 1), com equidistância entre as curvas de nível de 20 m, do Instituto Brasileiro de Geografia e Estatística - IBGE.

O software Spring 4.3, desenvolvido pela Divisão de Processamento de Imagens do Instituto Nacional de Pesquisas Espaciais (INPE), foi utilizado para a integração das informações em um banco de dados geográficos e para a obtenção dos parâmetros morfométricos propostos por Horton (1945).

A rede fluvial foi hierarquizada de acordo com Strahler (1957), que eliminou a subjetividade da classificação proposta por Horton (1945). A metodologia adotada para a análise morfométrica da rede de drenagem da bacia hidrográfica do rio Caeté seguiu a proposta de Horton divulgada na literatura, principalmente nos trabalhos de Horton (1945), Strahler (1957 e 1964), Christofoletti (1980) e Zãvoianu (1985).
Na Tabela 2, expõem-se resumidamente os princípios básicos das quatro Leis de Horton (1945), isto é, Lei de Número de Canais, Lei de Comprimento de Canais, Lei de Declividade e Lei de Área da Bacia de Canais.

Os métodos empregados para a determinação da dimensão fractal da rede de drenagem da bacia hidrográfica do rio Caeté foram os que se utilizam das razões de Horton (Tabela 3) e do método Box-Caunting (Contagem de Caixas), descrito por Goodchild (1982).

Para empregar o método Box-Counting, é necessário produzir uma malha quadriculada projetada sobre a figura, no caso a rede de drenagem. Contam-se quantas quadrículas foram interceptadas pelas linhas da figura.

A dimensão fractal será dada pela equação 1 .

$$
D=\lim \frac{\log N}{-\log d}
$$

em que $d$ é o comprimento do lado da quadrícula, do Box-Counting; e

$N$ é o número total de quadrículas interceptadas pela rede de drenagem.

Em um gráfico log-log, plotam-se os valores de $d$ e $N(d)$, e obtém-se uma relação linear entre logd e $\log N(d)$ com inclinação de $-k_{0}$, ou seja, a equação 2 .

$$
\log N(d)=-k_{0} \log d+\log \mu
$$

A dimensão fractal é o valor representado por $k_{0}$.

É importante destacar que, de acordo com Tarboton et al. (1988 apud BUENO JUNIOR, 2002), a dimensão fractal de cursos fluviais (segmentos) ( $d f$ ) deve usar valores de 15 a 125 m para $d$ e para a dimensão fractal da rede de drenagem $(D f)$ de uma bacia hidrográfica, valores de 250 a $1.000 \mathrm{~m}$ na escala real. Assim, os valores de $d$ adotados para o cálculo de $d f$ foram 0,$5 ; 1,0 ; 1,5$; e 2,0 mm e para o cálculo de $D f, 3,0 ; 6,0 ; 10,0 ;$ e 20,0 mm, na escala de 1:50.000 das cartas topográficas do IBGE. A malha da quadrícula foi gerada utilizando-se o software ArcView $3.2^{a}$, sendo a contagem realizada automaticamente a partir de rotinas deste.

Tabela 1 - Cartas topográficas utilizadas. Table 1 - Used topographical maps.

\begin{tabular}{ccccc}
\hline MI & Índice de nomenclatura & Nome da folha & Editor & Escala \\
\hline $2908-1$ & SG.22-Z-D-IV-1 & Alfredo Wagner & IBGE & $1: 50.000$ \\
$2908-2$ & SG.22-Z-C-IV-2 & Rancho Queimado & IBGE & $1: 50.000$ \\
$2908-3$ & SG.22-Z-C-IV-3 & Bom Retiro & IBGE & $1: 50.000$ \\
$2908-4$ & SG.22-Z-C-IV-4 & Anitápolis & IBGE & $1: 50.000$ \\
\hline
\end{tabular}

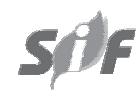

Revista Árvore, Viçosa-MG, v.34, n.4, p.661-668, 2010 
Tabela 2 - Resumo das Leis de Horton (1945).

Table 2 - Summary of the Horton's Laws (1945).

\begin{tabular}{|c|c|c|c|}
\hline Leis de Horton & Parâmetro Equação & Legenda & Observação \\
\hline $\begin{array}{l}\text { Lei do Número } \\
\text { de Canais }\end{array}$ & $\begin{array}{c}R_{b}=\text { taxa de } \\
\text { bifurcação } \\
R_{b}=\frac{N_{w}}{N_{w+1}}\end{array}$ & $\begin{array}{l}N_{w} \text { é o número de segmento } \\
\text { da ordem analisada. } N_{w+1} \text { é o } \\
\text { número de segmento da ordem } \\
\text { seguinte. }\end{array}$ & $\begin{array}{l}\text { O número de segmentos de ordens } \\
\text { sucessivamente inferiores de uma } \\
\text { bacia dada tende a formar uma } \\
\text { progressão geométrica, que começa } \\
\text { com o único segmento de ordem } \\
\text { mais elevada e cresce segundo uma } \\
\text { taxa constante de bifurcação. }\end{array}$ \\
\hline $\begin{array}{l}\text { Lei do comprimento } \\
\text { de Canais }\end{array}$ & $\begin{array}{l}R_{L}=\text { taxa de } \\
\text { comprimento } \\
R_{L}=\frac{\bar{L}_{w+1}}{\bar{L}_{w}}\end{array}$ & $\begin{array}{l}L_{w} \text { é o comprimento médio da } \\
\text { ordem analisada. } \\
L_{w+1} \text { é o comprimento médio dos } \\
\text { canais da ordem seguinte. }\end{array}$ & $\begin{array}{l}\text { O comprimento médio dos } \\
\text { segmentos de ordens sucessivos } \\
\text { tende a formar uma progressão } \\
\text { geométrica, cujo primeiro termo } \\
\text { é o comprimento médio dos } \\
\text { segmentos de primeira ordem e tem } \\
\text { por razão uma relação de } \\
\text { comprimento constante. }\end{array}$ \\
\hline $\begin{array}{l}\text { Lei da Declividade } \\
\text { de Canais }\end{array}$ & $\begin{array}{c}R_{s}=\text { taxa de } \\
\text { declividade média } \\
R_{s}=\frac{\bar{S}_{w}}{\bar{S}_{w+1}}\end{array}$ & $\begin{array}{l}S_{w} \text { é a declividade média da } \\
\text { ordem analisada. } S_{w+1} \text { é a } \\
\text { declividade média da ordem } \\
\text { seguinte. }\end{array}$ & $\begin{array}{l}\text { Em uma determinada bacia há uma } \\
\text { relação definida entre a declividade } \\
\text { média dos canais de certa ordem } \\
\text { e a dos canais de ordem } \\
\text { imediatamente superior, que pode } \\
\text { ser expressa por uma série } \\
\text { geométrica inversa, na qual o } \\
\text { primeiro termo é a declividade média } \\
\text { dos canais de primeira ordem e a } \\
\text { razão é a relação entre os gradientes } \\
\text { dos canais. }\end{array}$ \\
\hline $\begin{array}{l}\text { Lei da Área da } \\
\text { Bacia de Canais }\end{array}$ & $R_{a}=\frac{\bar{A}_{w+1}}{\bar{A}_{w}}$ & $\begin{array}{l}\overline{\mathrm{A}}_{\mathrm{w}} \text { é a área média das bacias de } \\
\text { cada canal da ordem analisada. }\end{array}$ & $\begin{array}{l}\text { As áreas médias das bacias de } \\
\text { segmentos de canais de ordem } \\
\text { sucessivos tendem a formar uma } \\
\text { progressão geométrica cujo } \\
\text { primeiro termo é a área média das } \\
\text { bacias de primeira ordem e a razão } \\
\text { de incremento constante é a taxa } \\
\text { de área. }\end{array}$ \\
\hline
\end{tabular}

Tabela 3 - Métodos empregados para estimar a dimensão fractal, a partir das Leis de Horton.

Table 3 - Methods used in the fractal dimension, starting from Horton's Laws.

\begin{tabular}{|c|c|c|}
\hline \multirow[t]{2}{*}{ Método } & \multicolumn{2}{|c|}{ Equações } \\
\hline & $d f$ & $D f$ \\
\hline Feder (1998) & $d f=2 \frac{\ln R_{L}}{\ln R_{B}}$ & \\
\hline Rosso et al. (1991) & $d f=\max \left(1,2 \frac{\log R_{L}}{\log R_{A}}\right)$ & $D f=\min \left(2,2 \frac{\log R_{B}}{\log R_{A}}\right)$ \\
\hline La Barbera e Rosso (1987 e 1989) & & $D f=\max \left(\frac{\log R_{B}}{\log R_{L}}, 1\right)$ \\
\hline Tarboton et al. (1990) & & $D f=d f \frac{\log R_{B}}{\log R_{L}}$ \\
\hline La Barbera e Rosso (1990) & & $D f=\left(\frac{1}{2-d f}\right) \frac{\log R_{B}}{\log R_{L}}$ \\
\hline
\end{tabular}

Fonte: SCHÜLLER et al., 2001.

Revista Árvore, Viçosa-MG, v.34, n.4, p.661-668, 2010 


\section{RESULTADOS E DISCUSSÕES}

A rede hierarquizada de drenagem da bacia hidrográfica do rio Caeté apresentou grandeza de $5^{\mathrm{a}}$ ordem. Os resultados das Leis de Horton são mostrados na Tabela 4. Os valores da $R_{b}, R_{L}$, Rs e $R_{a}$ mostraram-se válidos para a bacia hidrográfica, ou seja, entre os limiares aceitáveis, de acordo com Horton (1945) (Figura 2).
Os dados para a determinação da dimensão fractal pelo método de Box-Counting são mostrados na Tabela 5 e nas Figuras 3e 4. Ovalor de $d f$ encontrado para a bacia hidrográfica do Caeté foi de 1,0218 e o de $D f$, de 1,3828.

Diante dos valores de $d f$ e $D f$ obtidos pelo método de Box-Counting, verificou-se que eles semostraram satisfatórios, sendo $D f$ maior que $d f$. O primeiro retrata a rede de drenagem como um todo e o segundo, os segmentos individuais.

Tabela 4 - Resultados da aplicação das Leis de Horton na Bacia do Rio Caeté.

Table 4 - Results of the application of Horton's Laws in Caeté River watershed.

\begin{tabular}{cccccccccccccc}
\hline $\begin{array}{c}\text { Ordem } \\
(\mathrm{w})\end{array}$ & $\mathrm{Nw}$ & $\begin{array}{c}\log _{10} \\
\mathrm{Nw}\end{array}$ & $\mathrm{Rb}$ & $\begin{array}{c}\mathrm{Lw} \\
\text { médiam }\end{array}$ & $\begin{array}{c}\log _{10} \\
\mathrm{Lw}\end{array}$ & $\mathrm{R}_{\mathrm{L}}$ & $\begin{array}{c}\mathrm{Sw} \\
\text { média }\end{array}$ & $\begin{array}{c}\log _{10} \\
\mathrm{Sw}\end{array}$ & $\begin{array}{c}\text { Rs } \\
\text { Área }\end{array}$ & $\begin{array}{c}\text { Awmédia } \\
\mathrm{km}^{2}\end{array}$ & $\begin{array}{c}\log _{10} \\
\mathrm{Aw}\end{array}$ & $\begin{array}{c}\mathrm{Ra} \\
\mathrm{km}^{2}\end{array}$ \\
\hline $1^{\circ}$ & 252 & 2,40 & 4,3 & 718,3 & 2,9 & 1,6 & 0,194 & $-0,7$ & 1,4 & 92,40 & 0,4 & $-0,4$ & 4,4 \\
$2^{\circ}$ & 58 & 1,76 & 4,8 & 1126,3 & 3,1 & 1,9 & 0,135 & $-0,9$ & 1,4 & 92,56 & 1,6 & 0,2 & 4,2 \\
$3^{\circ}$ & 12 & 1,08 & 3,0 & 2122,4 & 3,3 & 2,2 & 0,099 & $-1,0$ & 2,3 & 80,70 & 6,7 & 0,8 & 4,3 \\
$4^{\circ}$ & 4 & 0,60 & 4,0 & 4698,9 & 3,7 & 3,3 & 0,042 & $-1,4$ & 5,5 & 115,84 & 29,0 & 1,5 & 5,7 \\
$5^{\circ}$ & 1 & 0,00 & & 15666,0 & 4,2 & & 0,008 & $-2,1$ & & 163,76 & 163,8 & 2,2 \\
Média & & & 4,0 & & & 2,3 & & & 2,7 & & \\
\hline
\end{tabular}

Nw é o número de canais; Rb é a taxa de bifurcação; Lw é o comprimento médio; $\mathrm{R}_{\mathrm{L}}$ é a taxa de comprimento; Sw é a declividade média; Rs é a taxa de declividade; Aw é área média; e Ra é a taxa de área.
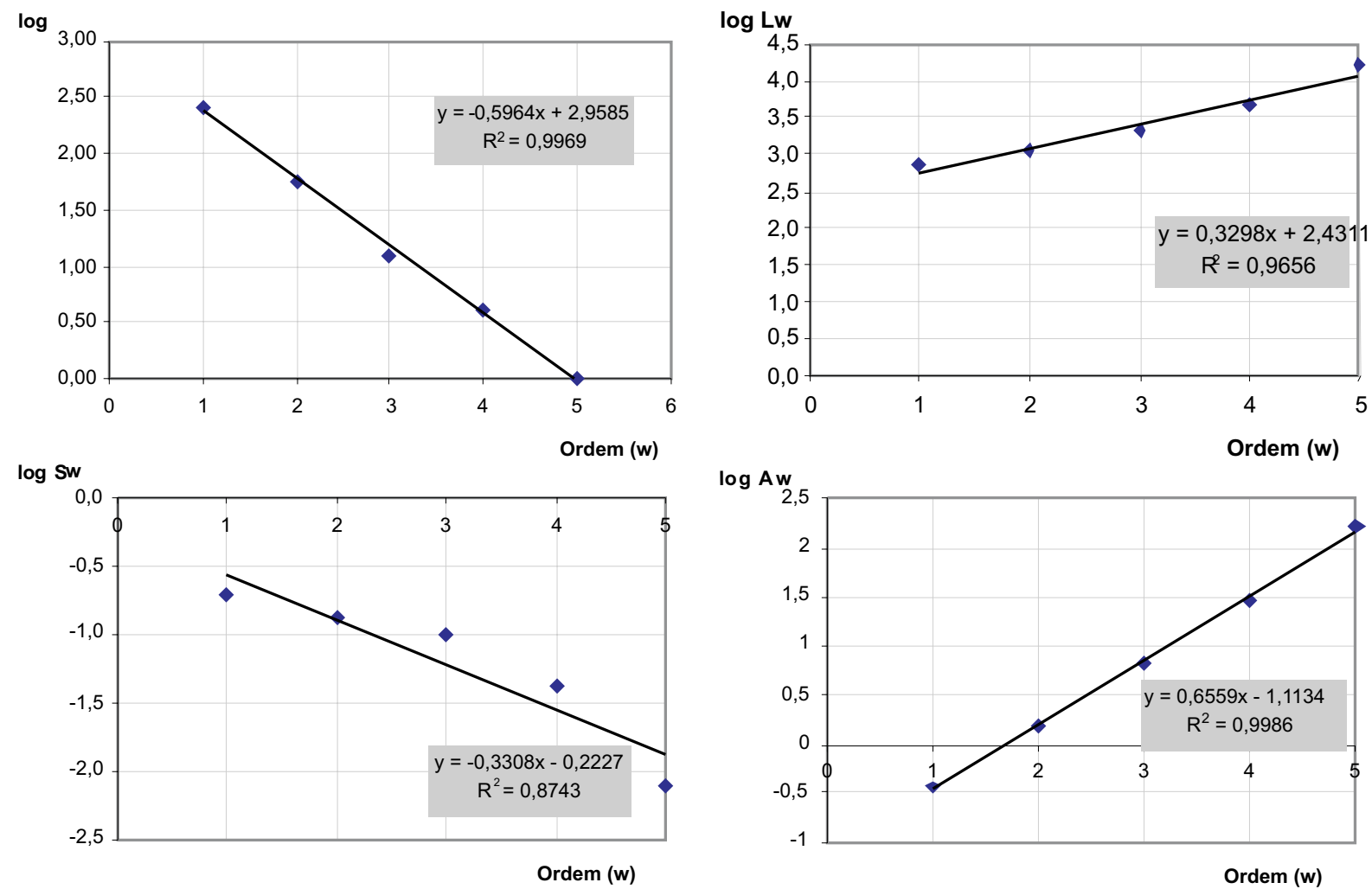

Figura 2 - Número de ordem (w) e o logaritmo do número de canais (Nw), do comprimento médio de canais (Lw), da declividade dos canais (Sw) e da área média das bacias de canais (Aw), na bacia hidrográfica do Caeté.

Figure 2 - Order number ( $w$ ) and the logarithm of the channels number $(N w)$, channels medium length (Lw), channels steepness ( $S w)$ and the basins channels medium area (Aw) of the Caeté River watershed. 
Tabela 5 - Valores de $d(\mathrm{~mm})$ e $N(\mathrm{~d})$ da bacia hidrográfica do Caeté.

Table 5 - Values $d(\mathrm{~mm})$ and $N(d)$ of the Caeté River watershed.

\begin{tabular}{cccc}
\hline$d(\mathrm{~mm})$ & $\log d(\mathrm{~mm})$ & $N(d)$ & $\log N(d)$ \\
\hline 20,0 & 1,301029996 & 182 & 2,260071388 \\
10,0 & 1,000000000 & 565 & 2,752048448 \\
6,0 & 0,778151250 & 1134 & 3,054613055 \\
3,0 & 0,477121255 & 2515 & 3,400537989 \\
2,0 & 0,301029996 & 3868 & 3,587486465 \\
1,5 & 0,176091259 & 5258 & 3,720820582 \\
1,0 & 0,000000000 & 7918 & 3,898615497 \\
0,5 & $-0,301029996$ & 16009 & 4,204364205 \\
\hline
\end{tabular}

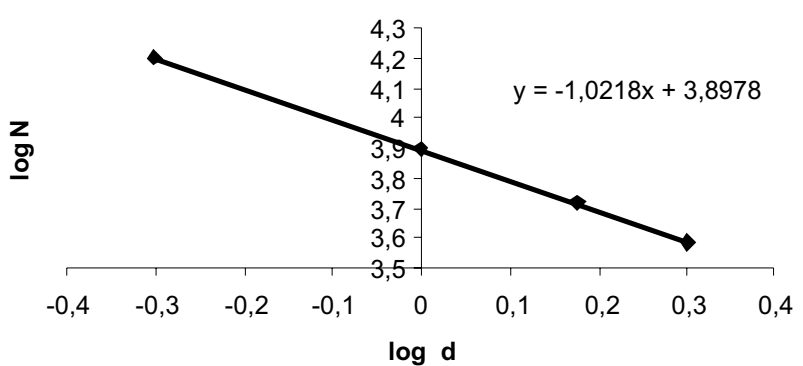

Figura 3 - Valores dos $\log$ de $d$ e $N(d)$, para $d f$. Figure 3 - Values of the log of $d$ and $N(d)$, for $d f$.

Na Tabela 6 são apresentadas as dimensões fractais obtidas a partir dos métodos que utilizam as Leis de Horton. Os resultados de $d f$ obtidos pelos três diferentes métodos mostraram valores mais divergentes do que os obtidos para $D f$, ou melhor, divergiram de 1,02 (Box-Counting) a 1,20 (FEDER, 1998). Sendo a $d f$ referente a segmento individual de drenagem, supõe-se que os valores teriam que ser próximos de 1,00; nesse caso, o valor obtido pelo método de Box-Counting mostrou-se mais próximo. No caso da $D f$, foi observado que os diferentes métodos, quando comparados, produziram resultados entre 1,66 e 1,81 .

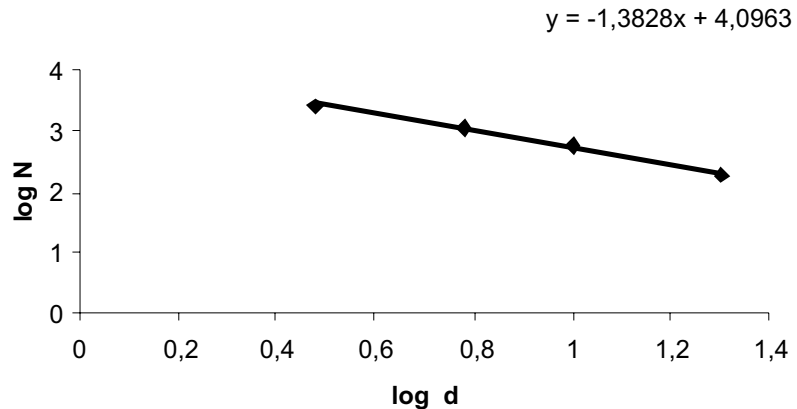

Figura 4 - Valores dos $\log$ de $d$ e $N(d)$, para $D f$. Figure 4 - Values of the log of $d$ and $N(d)$, for $D f$.

Os altos valores da $D f$ obtidos por meio das razões de Horton indicam que a rede de drenagem da bacia hidrográfica do rio Caeté apresenta elevado grau de complexidade, uma vez que os valores da $D f$ variam entre 1,00 e 1,99, na rede de drenagem.

Os valores da dimensão fractal obtidos pelos diferentes métodos mostraram-se coerentes com a teoria fractal, ou seja, comprovaram a existência de autossimilaridade e de multiescalonamentona redededrenagem dabaciahidrográfica do rio Caeté.

A densidade da rede de drenagem indica o grau de desenvolvimento do sistema de drenagem de uma bacia hidrográfica, por isso subsidia seu planejamento

Tabela 6 - Dimensão fractal estimada para a rede hidrográfica da bacia do Caeté.

Table 6 -Dimension fractal of the drainage network of the Caeté River watershed.

\begin{tabular}{lcc}
\hline Método & Equações \\
\cline { 2 - 3 } & \multicolumn{1}{c}{$d f$} \\
Feder (1998) & 1,201634 & 1,816833 \\
Rosso et al. (1991) & 1,091584 & 1,664401 \\
La Barbera \& Rosso (1987 e 1989) & 1,700684 \\
Tarboton et al. (1990) & & 1,701493 \\
La Barbera \& Rosso (1990) & & 1,793 \\
\hline
\end{tabular}

O valor da $d f$ empregada para cálculo da $D f$ foi de 1,0218, obtida pelo método de Box-Counting.

Revista Árvore, Viçosa-MG, v.34, n.4, p.661-668, 2010 
de manejo, como destacaram Cardoso et al. (2006). Assim, quanto maior a dimensão fractal, maior a complexidade apresentada por essa rede de drenagem.

A dimensão fractal manifesta a regularidade escalar da rede de drenagem, pois o conjunto espacial manifesta uma relação escalar, entre o número de seus elementos constituintes e a sua classe de mensuração (tamanho, densidade e intensidade), eliminando, assim, o problema da escala de representação (resolução) dos mapas.

\section{CONCLUSÃO E CONSIDERAÇÕES}

A $D f$ da rede de drenagem da bacia hidrográfica do Caeté, estimada pelos diferentes métodos, apresentou-se entre 1,66 e 1,81, enquanto a $d f$ ficou entre 1,02 e 1,20. Ambos os métodos utilizados apresentaram valores dentro do esperado pela geometria fractal.

Os métodos para a determinação da dimensão fractal mostraram-se de fácil aplicação.

Uma avaliação mais consistente sobre os métodos não foi possível pela inexistência de parâmetros fixos de referência.

A geometria fractal deve ser avaliada em novos estudos que visem comparar sistemas de drenagem diferenciados, visando estabelecer dimensões fractais que os representem.

\section{REFERÊNCIAS}

AGNESE, C.; D’ASARO, F.; GROSSI, G. ROSSO, R. Scaling properties of topologically random channel networks. Journal of hydrology. n.187, p. 183-193, 1996.

BUENO JUNIOR, J. Análise morfométrica da bacia do Rio Marumbi-PR, baseada nas leis de Horton e na Geometria Fractal. Curitiba: UFPR, 2002. p.53 (Monografia do Departamento de Geografia da UFPR).

CARDOSO, C. A.; DIAS, H. C. T.; SOARES, C. P. B.; MATINS, S. V. Caracterização morfométrica da bacia hidrográfica do Rio Debossan, Nova Friburgo, RJ. Revista Árvore. Viçosa-MG, v. 30, n. 2, p.241-248, 2006.

CHECCHIA, T.; KOBIYAMA, M.; SILVA, R. V.; ALVES, A. Análise preliminar da evolução dos deslizamentos no vale do Rio Caeté, Alfredo Wagner, SC In: Simpósio Nacional de Geomorfologia, 5., 2004, Santa Maria. Anais... Santa Maria: Universidade Federal de Santa Maria, 2004. 1 CD-ROM
CHRISTOFOLETTI, A. Geomorfologia. São Paulo: Ed. Edgard Blucher, 1980.

CHRistofoletti, A. L. H. Análise Fractal e Multifractal de Estações Chuvosas em Localidades do Estado de São Paulo. São Paulo, 1997. 263 f. Tese de Doutorado - UNESP/ Rio Claro.

GOODCHILD, M. F. The fractal Brownian processes as a terrain simulation model. Modeling and Simulation, Pittsburgh, v.13, p. 1133-1137,1982.

HORTON, R. E. Erosional development of streams and their drainage basins: a hydrophysical approach to quantitative morphology. Geol Soe. Am. Bull., v.56, n.3, p.275-370, 1945.

KOBIYAMA, M.; BUENO JUNIOR, J. Multifractal characteristics of drainage network of Marumbi river watershed, Paraná state. In: XXXI Congresso Brasileiro de Engenharia Agrícola, Salvador, 29 de julho a 02 de agosto de 2002. p. 432-435.

MANDELBROT, B. B. The fractal geometry of nature. San Francisco: W.H. Freeman and Co., 1983. 468p.

NIKORA, V. I. On self-similarity of drainage basins. Water Resources Research. v. 30, n. 1, p. 133-137, 1994.

PUENTE, C. E.; CASTILLO, P. A. On the fractal structure of networks and dividers within a watershed. Journal of hydrology. n.187, p. 173-181, 1996.

ROTH, G.; LA BARBERA, P.; GRECO, M. On the description of the basin effective drainage structure. Journal of hydrology. n.187, p. 119-135, 1996.

SANTA CATARINA. Gabinete de Planejamento e Coordenação Geral. Atlas do estado de Santa Catarina. Rio de Janeiro. 1986. 173p.

SCHÜLLER, D. J.; RAO, A. R.; JEONG, G. D.

Fractal characteristics of dense stream networks. Journal of hydrology. n.243, p. 1-16, 2001.

Revista Árvore, Viçosa-MG, v.34, n.4, p.661-668, 2010 
SILVA, R. V.; KOBIYAMA. M. Mapeamento de erosão potencial de encostas por meio de delineamento automático do escoamento superficial. In: Simpósio Brasileiro de Desastres Naturais, 1., 2004, Florianópolis. Anais... Florianópolis: Universidade Federal de Santa Catarina. 2004. p. 129-143. 1 CD-ROM

STRAHLER, A. N. Quantitative analysis of watershed Geomorphology. Am. Geophys. Union Trans. 38 (6): 913-920, 1957.

STRAHLER, A. N. Quantitative geomorphology of drainage basins and chanel networks. In:

CHOW, V. T. (ed.). Handbook of applied hydrology. New York: Mc-Graw-Hill, 1964.

TARBOTON, D. G. Fractal river networks, Horton's laws and Tokunaga cyclicity. Journal of hydrology. n.187, p. 105-117, 1996.

TARBOTON, D. G.; BRAS, R. L.; RODRIGUEZITURBE, I. The fractal nature of river networks. Water Resources Research 24 (8) 1317-1322, 1988.
TONELLO, K. C.; DIAS, H. C. T.; SOUZA, A. L.; RIBEIRO, C. A. A. S.; LEITE, F. P. Morfometria da bacia hidrográfica da Cachoeira das Pombas Guanhães - MG. Revista Árvore. ViçosaMG, v. 30, n. 5, p.849-857, 2006.

VIECILI, F. L. Mapeamento temático do uso e cobertura do solo na bacia do Caeté, município de Alfredo Wagner, SC. Florianópolis, 2005. 197f. Dissertação (Mestrado em Engenharia Ambiental) Universidade Federal de Santa Catarina.

VELTRI, M.; VELTRI, M.; MAIOLO, M. On the fractal description of natural channel networks. structure. Journal of hydrology. n.187, p. 137-144, 1996.

VESTENA, L. R.; CHECCHIA, T.; KOBIYAMA, M. Análise morfométrica da bacia hidrográfica do Caeté, Alfredo Wagner/SC. In: VI SIMPÓSIO NACIONAL DE GEOMORFOLOGIA REGIONAL CONFERENCE ON GEOMORPHOLOGY, 6., 2006, Goiânia. Anais/Actes... Volume II. Goiânia: União da Geomorfologia Brasileira / International Association of Geomorphologists, 2006. 1 CD-ROM

ZÃVOIANU, I. Morphometry of drainage basins. New York: Elsevier Science Publisher, 1985. 